\title{
Towards In-service Acoustic Characterization of Gaseous Microbubbles Applied to Liquid Sodium
}

\author{
M. Cavaro, J. Moysan, G. Corneloup, C. Gueudré, C. Payan, F. Baqué, G. Prele, J. Sibilo
}

\begin{abstract}
The characterization of argon microbubbles in liquid nitrogen is currently being investigated in connection with the development of Generation IV nuclear reactors. It seems that non-linear acoustic techniques can be used to determine the void fraction (volumetric fraction of free gas) and the histogram of the bubble radiuses. We discuss the first stage of our work here: the installation of an experimental test bench suitable for generating air microbubbles in water and then measuring them optically, before attempting an acoustic characterization. The bubble clouds generated have a radius distribution centred on about 20 microns.
\end{abstract}

Index Terms-Nonlinear acoustics - Microbubble cloud Liquid sodium

\section{INTRODUCTION}

L iquid sodium-cooled nuclear reactors (SFR) are among the serious candidates for the development of Generation IV reactors.

The continuous development this type of reactor is a major R\&D guideline today. Within this context, we are currently seeking to develop acoustic methods for characterizing argon in the form of free gas bubbles in liquid sodium.

The MESANGE bench has been developed to do this, which is used to generate a cloud of gas microbubbles whose properties are similar to those of the gas into liquid metal encountered in a reactor. This cloud bubble is accordingly characterized by a camera, which provides us with a reference to help develop and validate acoustic techniques for measuring the void fraction and the cloud bubble radius histogram. The acoustic techniques considered are non-linear methods: the mixing of two high frequencies and non-linear resonant ultrasonic spectroscopy (NRUS).

F.Baqué (e-mail: francois.baque@cea.fr) is with CEA Cadarache, DEN/DTN/STPA/LTTS, Bât. 201, 13108 St Paul lez Durance CEDEX, France

M.Cavaro is with CEA Cadarache, DEN/DTN/STPA/LIET, Bât. 201, 13108 St Paul lez Durance CEDEX, France

J. Moysan (e-mail: joseph.moysan@univmed.fr) and G. Corneloup (email : gilles.corneloup@univmed.fr) work for the Laboratoire de Caractérisation Non Destructive (LCND) - Université de la Méditerranée, Aix en Provence, 13625 France.

J.Sibilo is with AREVA-NP, 10 rue Juliette Récamier 69456 Lyon CEDEX 06, France
The industrial issues are described here, along with the MESANGE experimental bench, our first optical measurements, and the acoustic techniques considered.

\section{INDUSTRIAL ISSUES}

\section{A. Inspectability of objects immersed in sodium}

SFR-type reactors have the particular feature of using liquid sodium as a coolant. This fluid phase, present in the primary reactor vessel, flows through the core, the pumps and the heat exchangers in order to remove the heat produced by fission. Liquid sodium is opaque which complicates inspection. Methods based on acoustic wave propagation are therefore preferable in such an environment. The propagation of mechanical waves in a two-phase fluid is highly disturbed in comparison with the single-phase case. In fact, the liquid sodium of an SFR reactor is gassed continuously.

\section{B. Gas into Primary Sodium}

The sodium present in the main vessel, called primary sodium, is covered by a blanket gas, also referred to as the gas-cover plenum (generally argon). Ideally, this liquid sodium is perfectly pure and single-phase. However, this is not the case in the actual operating conditions: in addition to containing a few impurities and dissolved gases, free gas bubbles are continuously generated into liquid sodium. This paper will be focusing on this "gas into liquid metal" phenomenon. Studies conducted by the CEA have helped to identify the various sources of continuous gassing:

- The main source of the continuous formation of free gas in the primary sodium is the dissolution of gas in certain reactor areas, followed by nucleation (formation of free gas bubbles) which occurs in the sodium cooling zones, e.g. heat exchanger outlets, and/or where the hydrostatic pressure is decreased. In the case of argon, Henry's coefficient (solubility coefficient) in sodium is $2.64 \times 10^{-8} \mathrm{~mol}_{\mathrm{mol}}^{-1} \cdot \mathrm{bar}^{-1}$ at $550^{\circ} \mathrm{C}$ (hot plenum) but only $1.74 \times 10^{-9} \mathrm{~mol}_{\mathrm{mol}}^{-1} \cdot \mathrm{bar}^{-1}$ at $400^{\circ} \mathrm{C}$ (cold plenum) [1] with a hydrostatic pressure difference of about 0.7 bar between the two plenums. The approximate factor of nine between the two solubility values causes high supersaturation which is responsible for the generation of microbubbles nucleated during the flow of sodium in the reactor. A study has shown that most of the bubbles present in fast reactors are nucleated at the cold walls (outlet) of the intermediate heat exchangers. 
- Entrainment during the flow of sodium into the vessel's weir (if the reactor has one), causing a "waterfall effect".

- The appearance of a vortex at the free surface of the sodium when flow is turbulent. Since SFRs tend to be more compact, the flow will very likely be more turbulent, and thus the free surfaces will be more agitated if there is no anti-vortex device.

- Entrainment caused by the rotation of the submersible pump shafts.

- Gas production by neutron reactions (the appearance of gases such as neon, helium-3 or argon-41 due to the presence of impurities in the sodium).

- Return of the sodium cycled in outside auxiliary circuits, if any, and if they contain sources of gas.

- In certain cases, incidents such as small leaks can become sources of continuous gas into sodium.

\section{Consequences of Gas into Primary Sodium}

There was continuously gas into primary sodium of Phénix and Superphénix Plants (two French SFRs). And yet, neither two reactors had any instrumentation capable of characterizing this gassing. The very low void fractions involved never revealed any problems in terms of thermal and neutron core disturbances. In the case of Gen IV reactors, our viewpoint has changed for various reasons:

- The presence of bubbles in a liquid strongly alters its acoustic properties (celerity, attenuation, linearity, etc.). The application of acoustic measuring methods for continuous monitoring or periodic inspection (US telemetry, sonar, US thermometry, etc.) operating in rated conditions, hence with gas into sodium, requires approximate knowledge of the attenuation coefficients, to prove in principle that the signal amplitude will be sufficient, as well as approximate knowledge of the homogeneity defect in the spatial distribution, to prove that the sound velocity calibrations performed at some distance from the actual measurement point remain useable. This necessarily implies knowledge of the void fraction and of certain data concerning the bubble radius histograms (at least the bounds).

- If the void fraction evaluated by Superphénix is not directly detrimental in itself with regard to core operation, it is indirectly so if it helps generate gas pockets at the high points of the immersed structures. The characterization of continuous gas into sodium in a reactor could provide input data for tests or calculations of the formation and release of these gas pockets.

- Furthermore, measurements and tests would help validate the CEA models simulating bubble behaviour in a reactor, in order to address the problem of free gas in a reactor from the project stage.

- Continuous monitoring of the gas into sodium rate seems necessary for preventing the overrun of several thresholds:

The neutron disturbance threshold (basically too high to be reached in normal reactor operating conditions).
The saturation of systems for measuring activity in the cover-gas plenum (caused by salting out of ${ }^{41} \mathrm{Ar}$ from low void fractions: this threshold has been evaluated at a void fraction of $10^{-4}$ in association with the FBR 1500 project).

- Finally, measuring continuous gas into primary sodium was the subject of precise requests from the French Nuclear Safety Authority in 1994 and 1995 sent to the Superphénix operator. The same requests therefore appear to be inevitable for the future reactors.

\section{Feedback from Superphénix}

The operation of the Phénix and Superphénix reactors provided us with feedback that enables us to roughly estimate the quantities of the gas into sodium that can be expected.

In 1996, decay measurements of ${ }^{41}$ Ar activity in the cover-gas plenum after a rapid shutdown, associated with the study of argon transport in sodium, served to determine a fairly accurate value of the void fraction in the cold plenum: $2.56 .10^{-}$ 6 with an uncertainty factor of 2 . These studies also demonstrated that most of the gas into sodium was due to nucleation of the dissolved gases, with an average bubble radius of $10 \mu \mathrm{m}$. Moreover, a second population of bubbles produced by entrainment at the weir had an average radius of several tens of microns. The two-phase flow to be considered is therefore that of a flow with dispersed spherical bubbles.

Within the context of future reactors, the intention is to minimise the sources of gas into the primary sodium. However, it is very difficult to know today the extent to which the normal void fraction will be decreased. It is planned to conduct our experiments below the void fraction of $10^{-6}$.

\section{E. Characterization of Gas into Sodium}

For all the reasons listed above, we have been requested to develop acoustic techniques designed to measure:

- $\quad$ The void fraction (as a minimum).

- The bubble radius histograms (at least its bounds).

And this is required for a microbubble cloud such that:

- $\quad$ The bubble radius is between 10 and 100 microns.

- The void fraction is about $10^{-6}$ and if possible less.

\section{MESANGE BENCH}

\section{A. General Description}

Since liquid sodium is opaque, it was impossible to use a simple reference measurement. This is why the MESANGE ${ }^{1}$ bench is an essential device. This bench consists of a water tank, a system for generating bubble clouds, an optical measurement device in the cloud, and the acoustic instrumentation needed to develop the planned techniques.

\section{B. Microbubble Generation}

To generate a cloud of air microbubbles in water, we selected the aeroflotation technique. Initially used for wastewater filtration, this technique is based on Henry's law:

\footnotetext{
${ }^{1}$ MESure Acoustique de l'eNGazement en Eau (acoustic measurement of gas into water)
} 


$$
C_{S}=p \cdot H
$$

where $\mathrm{C}_{\mathrm{S}}$ is the dissolved gas concentration at saturation, $\mathrm{p}$ is the partial pressure of the gas considered above the liquid, and $\mathrm{H}$ is Henry's coefficient (coefficient of gas solubility in the liquid, related to temperature). Thus, air is pressurised (7 bar100 PSI) in a chamber containing water, and when saturation is reached (in a few minutes), the water enriched with dissolved gas is injected into the vessel at atmospheric pressure. This water is in a state of supersaturation, so that the nucleation of air microbubbles occurs.

Our device was obtained from Sciential Supply \& Equipment LLC $®$ (Bench-Scale Dissolved Air Flotation Kit) and consists of a vacuum pump connected at a compressor and a chamber (Fig. 1).

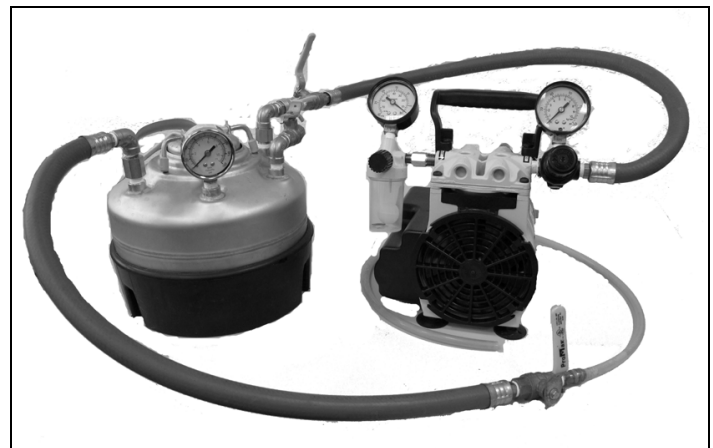

Fig. 1. Microbubble generation system based on aeroflotation

\section{Optical inspection of the bubble cloud}

Bubble nucleation mechanisms in supersaturated media are relatively complex because they are influenced by several parameters that are difficult to control, such as the quantity and size of the impurities inevitably present in the water. This is why it is necessary to perform optical measurements to obtain accurate knowledge of the radius histograms of the bubbles generated and the void fraction.

A monochrome digital camera $\left(\mathrm{JAI}{ }^{\circledR}-2456 \times 2058\right.$ pixels -15 $\mathrm{fr} / \mathrm{s})$ coupled with a telecentric optic (x1.5) was used to do this. The device is supplemented by a watertight chamber designed to immerse the camera and LED lighting for transmission.

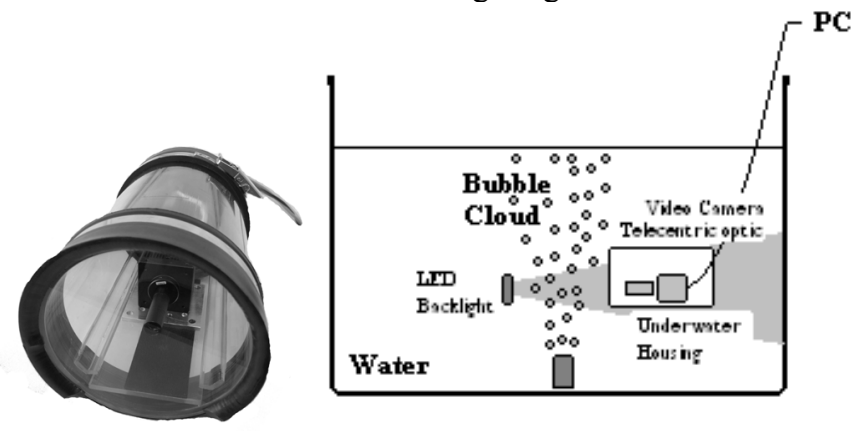

Fig. 2. Setup for submersible optical measurements

The resolution obtained is $2.3 \mu \mathrm{m} / \mathrm{pixel}$. The field observed by the camera is therefore about $5.6 \mathrm{~mm} \times 4.7 \mathrm{~mm}$. The depth of the optical field is announced at $0.66 \mathrm{~mm}$.

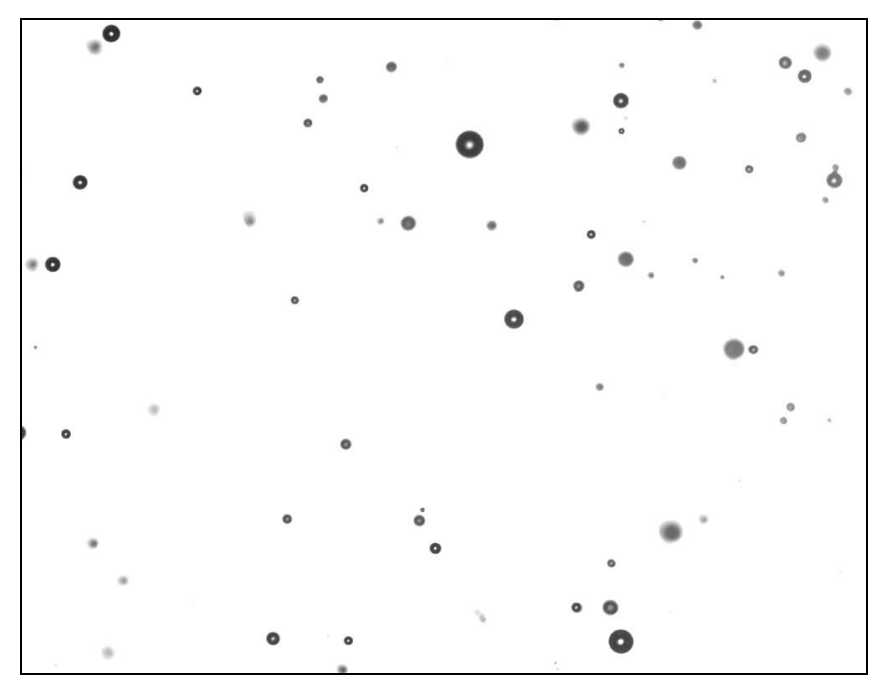

Fig. 3. Image of bubble clouds obtained from the optical device (field size $5.6 \mathrm{~mm} \times 4.7 \mathrm{~mm}$ )

The images produced therefore display more or less sharp bubbles (Fig. 3). Moreover, the bubbles have a white spot at their centre, corresponding to a passage of light (Fig. 4). Image processing is therefore necessary to obtain the histogram of the bubble radiuses.

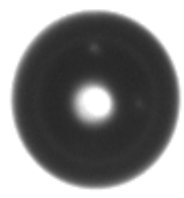

Fig. 4. Enlargement of a bubble

The image processing that we developed with the Matlab ${ }^{\circledR}$ software consists of image thresholding followed by bubble filling in order to overcome the problems raised by the presence of the central white spot. The bubbles are then labelled and their radius is calculated. The histogram of each image is then accessible and a calculation on a large number of images serves to statistically reconstruct the histogram representing the bubble population.

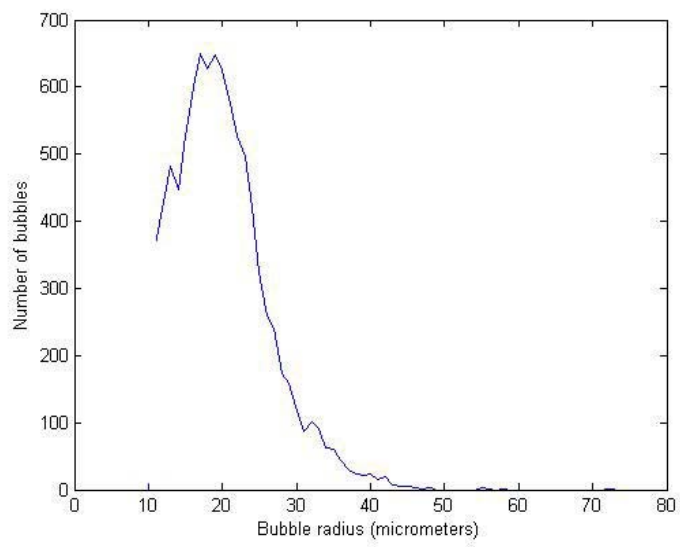

Fig. 5. Bubble radius histogram calculated on 1000 images 
The main difficulty of image processing based on thresholding is in the definition of this threshold. Figure 6 shows the histogram of the levels of a photograph. The first peak corresponds to the bubble population located at the working distance of the optics. The threshold to be applied to select this population is therefore easy to determine here. On a collection of images, the peaks are less sharp and considerable work is required on the stability of the lighting used in order to determine a reliable threshold.

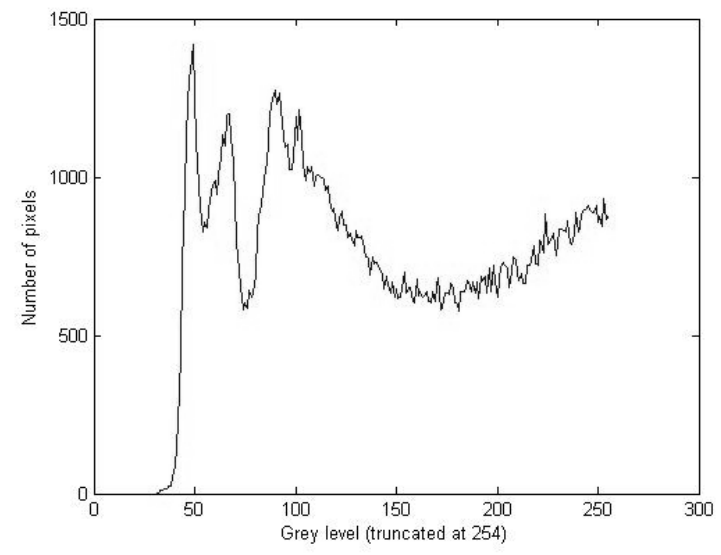

Fig. 6. Histogram of grey levels of an image

According to our first experiments, the bubble clouds generated after saturation at 7 bar and expansion at low flow rate display bubble radius histograms that are fairly constant and similar to the one shown in Figure 5. Changes in saturation pressure and flow rate should enable us to observe bubble populations with different properties: shift of average radius and/or spreading of the histograms.

\section{Acoustic Characterization of A BubBle Cloud}

\section{A. Choice of Acoustics}

Several methods of non-destructive characterization can be used in two-phase fluid media to characterize them. These are mainly:

- Techniques based on the electrical (resistance, capacitance) or magnetic (induction, NMR nuclear magnetic resonance) properties of the medium

- Optical techniques (optical fibres)

- Mechanical techniques (differential pressure measurements, samplings, etc.)

- Techniques based on ionising radiation (X-rays, $\gamma$, neutrons)

- Acoustic techniques.

Upon consideration of sodium properties (optical, electrical and chemical) and the very low volumetric density of the gases present, it appeared that acoustic techniques are the only methods feasible for our problem.

Acoustic techniques can be classed into two groups: linear techniques and non-linear techniques.

\section{B. Linear Acoustic Techniques}

For nearly a century, the acoustics of two-phase media have been the subject of various investigations [2], [3], [4], [5]. Linear acoustic techniques have been proposed for several decades for the characterization of bubble sizes and densities of two-phase liquid-gas media: measurement of attenuation, backscattering, celerity, etc.

Figure 7 clearly shows the main limitation to the use of linear acoustic techniques. A given diffusion cross-section corresponds to two cases: a resonant bubble or a non-resonant but much larger bubble. This ambiguity proves to be insoluble in every case where the size distribution of the bubble population is unknown. This is basically the case in a reactor.

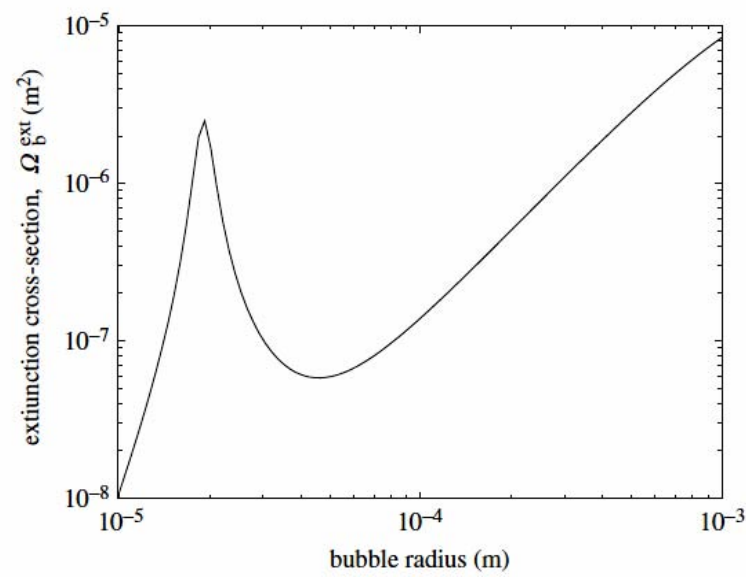

Fig.7. Extinction cross-section for a fixed frequency of $165 \mathrm{kHz}$ as a function of bubble radius (from [14])

To deal with this ambiguity, we have to consider the use of non-linear acoustic techniques.

\section{Non-linear Acoustic Techniques}

Resonance mechanisms of a gas bubble in a liquid are highly non-linear. However, using these non-linearities is a much more recent approach than the linear approach to bubble behaviours [6].

One of the main equations governing the bubble dynamics, the Rayleigh equation (1917), used for decades in linear acoustics, is however essentially non-linear. The so-called modified Rayleigh-Plesset equation has the form:

$\rho R \ddot{R}+\frac{3}{2} \rho \dot{R}=\left(p_{0}+\frac{2 \sigma}{R_{0}}-p_{v}\right)\left(\frac{R_{0}}{R}\right)^{3 \gamma}-\frac{2 \sigma}{R}-\frac{4 \mu}{R} \dot{R}+p_{v}-p_{0}-P(t)$

where $\rho$ is the density of the liquid, $R$ the instantaneous radius of the bubble, $\dot{R}$ the bubble speed at the wall, $\ddot{R}$ its acceleration, $p_{0}$ the position at rest in the liquid, $p_{v}$ the liquid vapour pressure, $\sigma$ the surface tension, $R_{0}$ the bubble equilibrium radius, $\gamma$ the polytropic coefficient of the gas, $\mu$ the liquid viscosity and $P(t)$ the acoustic pressure.

The acoustically non-linear character of a propagation medium is illustrated in various ways:

- the generation of harmonics and subharmonics [7] 
- the mixing of two frequencies: when non-linear objects are simultaneously insonified by two waves of different frequencies $f_{1}$ and $f_{2}$, waves of difference frequencies $f_{1}-f_{2}$ and summation frequencies $f_{1}+f_{2}$ appear [8]

- non-linear resonant ultrasonic spectroscopy (NRUS) i.e. observing the frequency shift of the resonance as a function of the incident wave amplitude [9].

While the acoustic characterization of gas bubbles in a liquid has been the subject of many investigations for decades, few experiments have been validated on bubble clouds in order to characterize them. For this purpose, two non-linear techniques have drawn our attention today: the mixing of two high frequencies and NRUS.

The mixing of two high frequencies was tested by [8] on a bubble cloud. The inversion proposed serves to reconstruct a bubble radius histogram corresponding to the nonlinear responses observed. However, these experiments were not validated by a reference measurement. Experiments have also been conducted more recently at sea [10].

Non-linear resonance ultrasonic spectroscopy is a technique derived from the non-destructive characterization of solids such as bones [9] and concrete [11]. [12] shows that the theoretical behaviour of the bubbles make this technique useable in a two-phase medium, and [13] simulated the resonant behaviour of a bubble for several amplitudes (Fig. 8). We believe that the frequency shift observed in this model confirms the potential of NRUS to characterize a bubble cloud.

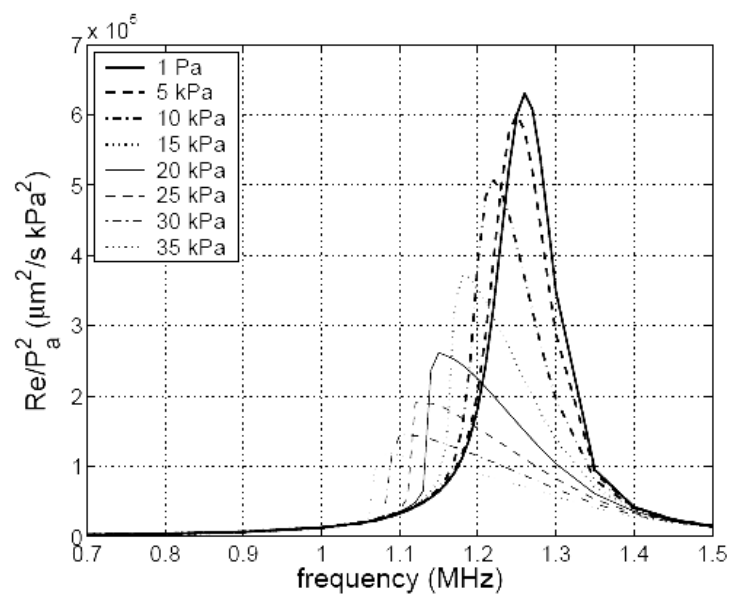

Fig. 8. Constructed power spectra by microbubble spectroscopy for different driving pressure amplitudes $(\mathrm{r}=3 \mu \mathrm{m})$ (from [13])

To the best of our knowledge, NRUS has never been tested on a bubble cloud.

\section{Acoustic Experiments}

The MESANGE bench should enable us to develop and validate the above two techniques: mixing of two high frequencies and NRUS.

The set-up installed to validate the frequency mixing is based on the set-up used in Reference [8]. (Fig.9)

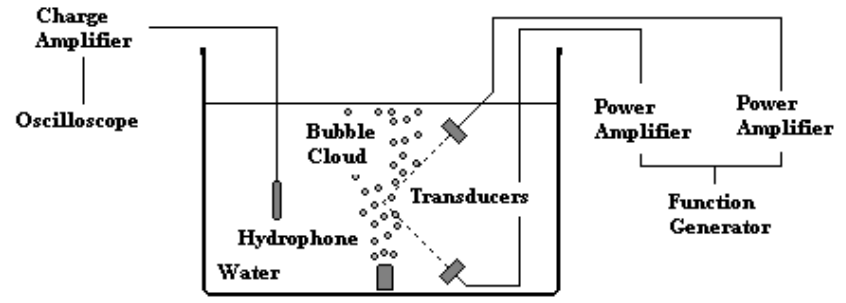

Fig. 9. Schematic experimental set-up based on reference [8]

The configuration(s) enabling us to test techniques based on NRUS are currently being developed. It is being considered to make a two-phase fluid resonate between two metal plates.

\section{PRELIMINARY RESUlts}

For the time being, only preliminary experiments have been conducted. The insonification of our bubble clouds at various amplitudes reveals a frequency shift in the cloud response, which is encouraging.

\section{FUtURE PROSPECTS}

The conclusive experiments conducted by reference [8] lead us to believe that they will be validated and, in particular, the inversion proposed with our optical set up in the medium term.

If not, other theoretic inversions of non-linear acoustic behaviour of a bubble cloud ([14] for example) provide other alternatives that can be tested and validated experimentally on the MESANGE bench.

As part of the use of NRUS, it is possible that an attempt will be made in the medium term to apply models derived from the characterization of solids, in order to determine the bubble fractions and the bubble radius histogram [11], [15].

\section{REFERENCES}

[1] E. Honnorat - Propriétés physiques de l'argon à l'état gazeux - Recommendations CEA No13, 03/1978

[2] A. Mallock - The damping of sound by frothy liquids Proceedings of the Royal Society of London. Series A, Containing Papers of a Mathematical and Physical Character, Volume 84, Issue 572, pp. 391-395

[3] K.W. Commander, A. Prosperetti - Linear pressure waves in bubbly liquids: comparison between theory and experiments - The Journal of the Acoustical Society of America, 85(2), p. 732 à 74, Feb. 1989

[4] T.G. Leighton - The Acoustic Bubble - Academic Press, 1994

[5] T.G. Leighton - From seas to surgeries, from babbling brooks to baby scans : the acoustics of gas bubbles in liquids - International Journal of Modern Physics B, Vol.18, N.25, p.3267-3314, 2004 
[6] L. Bjorno - Forty years of nonlinear ultrasound Ultrasonics 40, p.11-17, 2002

[7] S. Kumar, C.E. Brennen - Nonlinear effects in the dynamics of clouds of bubbles - J. Acous. Soc. Am., Vol. 89(2), p. 707-714, Feb. 1991

[8] A.M. Sutin et al. - Nonlinear acoustic method for bubble density measurements in water - J. Acous. Soc. Am., Vol. 103(5), p. 2377-2384, May 1998

[9] M. Muller et al. - Nonlinear resonant ultrasound spectrosopy (NRUS) applied to damage assessment in bone J. Acous. Soc. Am., Vol. 118(6), p. 3946-3952, Dec. 2005

[10] L.A. Ostrovsky et al. - Nonlinear scattering of acoustic waves by natural and artificially generated subsurface bubble layers in sea - J. Acous. Soc. Am., Vol. 113(2), p. 741-749, Feb. 2003

[11] C. Payan - Caractérisation non destructive du béton: étude du potentiel de l'acoustique non linéaire - Mémoire de doctorat, Université de la Méditerranée, 2007
[12] A. Prosperetti - Nonlinear oscillations of gas bubbles in liquids : steady state solutions - - J. Acous. Soc. Am., Vol. 56(3), p. 878-885, Sep. 1974

[13] S. Van Der Meer - Ultrasound Contrast Agents. Resonances of coated bubbles - Thesis, Physics of Fluids, University of Twente

[14] T.G. Leighton, S.D. Meers, P.R. White - Propagation through nonlinear time-dependent bubble clouds and the estimation of bubble populations from measured acoustic characteristics - Proc. R. Soc. Lond. A - 460, 2521-2550, 2004

[15] K. Van Den Abeele, F. Windels - Characterization and Imaging of Microdamage Using Nonlinear Resonant Ultrasound Spectroscopy (NRUS): An Analytical Model Book chapter of Universality of Nonclassical Nonlinearity, Springer New York, 2006

M. Cavaro is a young engineer from French ICAM (Institut Catholique d'Arts et Métiers, in Toulouse) College. He is now performing a $\mathrm{PhD}$ work on gas detection within liquid sodium. 